\title{
Remarks on Azarov's work on soluble groups of finite rank
}

\author{
B. A. F. Wehrfritz ${ }^{1}$
}

Received: 11 September 2015 / Accepted: 10 December 2015 / Published online: 9 January 2016

(C) The Author(s) 2016. This article is published with open access at Springerlink.com

\begin{abstract}
We present proofs of D. N. Azarov's recent three theorems determining precisely when a soluble group of finite rank is residually a finite $\pi$-group for a specified finite set $\pi$ of primes. Our proofs seem to be substantially shorter; they also apply to groups with a somewhat weaker notion of finite rank.
\end{abstract}

Keywords Soluble group · Finite rank · Residually finite

\section{Mathematics Subject Classification $20 \mathrm{~F} 16 \cdot 20 \mathrm{E} 26$}

According to its English summary Azarov's paper [1] is devoted to proving the following. If $\pi$ is a finite set of primes, then a soluble group of finite rank is a finite extension of a residually a finite $\pi$-group if and only if it is a finite extension of a residually finite nilpotent $\pi$-group, which happens if and only if it is a reduced FATR group with no $\pi$-divisible elements of infinite order (see below or see [3] for definitions). Further he proves that a soluble group of finite rank is residually a finite $\pi$-group for some finite set $\pi$ of primes if and only if it is a reduced FATR group. (This is effectively an old result of D. J. S. Robinson, see [3] 5.3 .8 or [4] Page 138.) We give below what presumably are much shorter proofs of these interesting results. In fact we prove the following. (Below $\tau(\mathrm{G})$ denotes the maximal periodic normal subgroup of a group G, FittG its Fitting subgroup and $\left\{\zeta_{\alpha}(\mathrm{G})\right\}$ its upper central series.)

Theorem Let $G$ be a finite extension of a soluble FAR group and let $\pi$ be some finite set of primes. The following are equivalent.

(a) $G$ is a finite extension of a residually finite $\pi$-group.

(b) $\tau(G)$ is finite and $\zeta_{1}($ FittH) is $\pi$-reduced for some normal subgroup $H$ of $G$ of finite index.

(c) $G$ is a finite extension of a residually finite nilpotent $\pi$-group.

B. A. F. Wehrfritz

b.a.f.wehrfritz@qmul.ac.uk

1 School of Mathematical Sciences, Queen Mary University of London, London E1 4NS, England 
(d) $G$ is a finite extension of a reduced soluble FATR group with no $\pi$-divisible elements of infinite order.

Corollary Suppose G is a finite extension of a soluble FAR group. The following are equivalent.

(a) There exists a finite set $\pi$ of primes such that $G$ is residually a finite $\pi$-group.

(b) $G$ is reduced and a finite extension of an FATR group.

(c) $\tau(G)$ is finite and $\zeta_{1}($ FittG) is reduced.

Soluble FAR and FATR groups are defined in [3]. An equivalent definition, often more convenient, is the following. A soluble group $G$ is FAR if it has finite Hirsch number and satisfies min-p for every prime p. (A group $G$ has Hirsch number $h$ if $G$ has a series of finite length with exactly $h$ of the factors infinite cyclic, the remaining factors of the series being locally finite; G satisfies min-p if it satisfies the minimal condition on p-subgroups.)

It is elementary that to within some normal subgroup of finite index, locally finite factors can essentially be moved down a series past torsion-free abelian factors of finite rank and finite factors can be moved up past torsion-free abelian factors of finite rank and past periodic abelian factors satisfying min-p for all primes $\mathrm{p}$. Further divisible abelian factors in a periodic FAR group sink to the bottom (e.g. [2] 3.18). Thus it is elementary to see that a group $\mathrm{G}$ is a finite extension of a soluble FAR group if and only if it has a characteristic series

$$
\langle 1\rangle=G_{0} \leq G_{1} \leq \cdots \leq G_{r} \leq \cdots \leq G_{s} \leq G,
$$

where $G_{1}$ is periodic, divisible, abelian and satisfies min-p for all primes $p, G_{i+1} / G_{i}$ for $1 \leq$ $i<r$ is infinite periodic abelian with all its primary components (i.e. its Sylow subgroups) finite, $\mathrm{G}_{i+1} / \mathrm{G}_{i}$ for $r \leq i<\mathrm{s}$ is torsion-free abelian of finite rank and $\mathrm{G} / \mathrm{G}_{s}$ is finite. The soluble FATR groups are exactly those $\mathrm{G}$ above with $\mathrm{G}_{1}$ involving only finitely many primes, with $\mathrm{r}=1$ and, of course, with $\mathrm{G} / \mathrm{G}_{s}$ soluble.

Suppose $\mathrm{G}$ is a finite extension of a soluble FAR group with its maximal periodic normal subgroup $\tau(\mathrm{G})$ finite. Then from the above, the following hold.

(a) $\mathrm{G}$ is (torsion-free)-by-finite and

(b) $\mathrm{G}$ is a finite extension of a soluble FATR group. Further

(c) FittG is nilpotent and G/FittG is abelian-by-finite (see [3] 5.2.2) and

(d) G/FittG is a finite extension of a free abelian group of finite rank (see [3] 5.2.3).

Note that in general FittG ${ }_{s}=\mathrm{G}_{s} \cap$ FittG, FittG/FittG is finite and FittG is nilpotent if and only FittG $_{s}$ is nilpotent. Also the soluble groups $\mathrm{G}$ of finite rank discussed by Azarov in [1] are exactly FAR groups above with $\mathrm{G} / \mathrm{G}_{s}$ soluble and $\mathrm{G}_{r}$ of finite rank.

\section{The proofs}

We use the following elementary results.

Lemma 1 Let $A$ be a torsion-free abelian group.

(a) If $\pi$ is any set of primes then $A$ is residually a finite $\pi$-group if and only if $A$ is $\pi$-reduced.

(b) If $A$ is reduced and of finite rank, then for some finite set $\pi$ of primes, $A$ is residually a finite $\pi$-group.

For example see [3] 5.3.4 and 5.3.5. We also use the following, see [4] 9.38. 
Lemma 2 Let $G$ be a nilpotent FAR group and $\pi$ any set of primes. Then $G$ is residually a finite $\pi$-group if and only if $\zeta_{1}(G)$ is $\pi$-reduced.

Thus in Lemma 2 if $\zeta_{1}(\mathrm{G})$ is $\pi$-reduced, then $\mathrm{G}$ is residually a finite $\pi$-group, so each $\mathrm{G} / \zeta_{i}(\mathrm{G})$ is also residually a finite $\pi$-group by Learner's Lemma. Hence each $\zeta_{i+1}(\mathrm{G}) / \zeta_{i}(\mathrm{G})$ is $\pi$-reduced, something that is easy to see directly.

The proof of the theorem (a) implies (b). Let $\mathrm{H}$ be a normal subgroup of $\mathrm{G}$ that is residually a finite $\pi$-group. Then $\zeta_{1}($ FittH) is $\pi$-reduced by Lemma 1. Also $\tau(\mathrm{H})$ is a $\pi$-group. If P is a p-subgroup of $\tau(\mathrm{H})$ then $\mathrm{P}$ is Chernikov, residually finite and hence finite, and $\pi$ is finite. Consequently $\tau(\mathrm{H})$ is finite. Clearly $\tau(\mathrm{G}) / \tau(\mathrm{H})$ is finite, so $\tau(\mathrm{G})$ is finite.

(b) implies (c). (This is actually the core of the proof of the Theorem.) There exist normal subgroups $\mathrm{N} \leq \mathrm{L}$ of $\mathrm{G}$ with $\mathrm{L} \leq \mathrm{H}, \mathrm{G} / \mathrm{L}$ finite, $\mathrm{L} / \mathrm{N}$ free abelian of finite rank and $\mathrm{N}=$ FittL torsion-free nilpotent of finite rank. Clearly $\mathrm{N} \leq$ FittH. By (b) and Lemma 2 FittH is residually a finite $\pi$-group, so $\mathrm{N}$ is residually a finite $\pi$-group.

Set $\mathrm{q}=\Pi_{p \in \pi} \mathrm{p}$ and $M=\cap_{i} C_{L}\left(\zeta_{i+1}(N)^{q} \zeta_{i}(N) / \zeta_{i}(N)\right)$. Clearly $\mathrm{N} \leq \mathrm{M} \leq \mathrm{L}$ and $\mathrm{G} / \mathrm{M}$ is finite. We claim that $\mathrm{M}$ is residually a finite nilpotent $\pi$-group. If so then c) holds. Now $\mathrm{M} / \mathrm{N}$ is free abelian, so $\mathrm{M} / \mathrm{N}$ at least is residually a finite nilpotent $\pi$-group.

Let $x \in N \backslash\langle 1\rangle$. Since $N$ is residually a finite $\pi$-group, there exists a power $m=q^{\mu}$ of $q$ with $\mathrm{x} \notin \mathrm{N}^{m}$. Now $\mathrm{N} / \mathrm{N}^{m}$ is finite and $\mathrm{M} / \mathrm{N}$ is polycyclic, so there exists a torsion-free normal subgroup $\mathrm{T} / \mathrm{N}^{m}$ in $\mathrm{M} / \mathrm{N}^{m}$ with $\mathrm{M} / \mathrm{T}$ finite. Also $\mathrm{N} / \mathrm{N}^{m}$ lies in the hypercentre of $\mathrm{M} / \mathrm{N}^{m}$ and $\mathrm{M} / \mathrm{N}$ is abelian. Hence $\mathrm{M} / \mathrm{N}^{m}$ is nilpotent, as therefore is its finite image M/T. Let $\mathrm{S} / \mathrm{T}$ denote the Hall $\pi$ '-subgroup of $M / T$. Then $M / S$ is a finite nilpotent $\pi$-group. Further $x \notin S$, since $x$ is a non-trivial $\pi$-element modulo $\mathrm{N}^{m}$ and $\mathrm{S} / \mathrm{N}^{m}$ is an extension of a torsion-free group by a $\pi^{\prime}$-group. It follows that $\mathrm{M}$ is residually a finite nilpotent $\pi$-group.

(c) implies (a). This is trivial. Thus (a), (b) and (c) are equivalent.

(a) and (b) imply (d). G is residually finite, so $G$ is reduced. Also $\tau(G)$ is finite, so $G$ is a finite extension of a residually finite- $\pi$, reduced FATR group H. Since H is residually finite- $\pi$, so $\mathrm{H}$ contains no $\pi$-divisible elements of infinite order. Consequently neither does G.

(d) implies (b). By (d) $\mathrm{G}$ has a reduced soluble normal FATR subgroup $\mathrm{H}$ of finite index. Then $\mathrm{H}$ is (torsion-free)-by-finite, $\tau(\mathrm{H})$ is finite and consequently $\tau(\mathrm{G})$ is finite. Further we may choose $\mathrm{H}$ torsion-free. Then $\mathrm{H}$ has no non-trivial $\pi$-divisible elements by (d) and hence $\zeta_{1}(\mathrm{FittH})$ is $\pi$-reduced. Thus (b) holds.

The proof of the corollary If (a) holds, then so does (b) by the Theorem. Clearly (b) implies (c). Suppose (c) holds. By Lemma 1 there exists a finite set $\kappa$ of primes such that $\zeta_{1}(\mathrm{FittG})$ is $\kappa$-reduced. Hence by the Theorem, (b) implies (a), there exists a normal subgroup $\mathrm{H}$ of $\mathrm{G}$ of finite index that is residually a finite $\kappa$-group. But then $\mathrm{G}$ is residually a finite $\pi$-group for $\pi=\kappa \cup\{$ all prime divisors of $(\mathrm{G}: \mathrm{H})\}$. Thus (a) holds.

Remark In a special but still quite general case there is a slicker but less elementary proof of (b) implies (c), the main implication of the theorem.

With $\mathrm{N}$ as in the original proof let $\mathrm{N}_{p}$ denote the finite-p residual of $\mathrm{N}$. Then $\cap_{p \in \pi} N_{p}=$ $\langle 1\rangle$. The upper central factors of $\mathrm{N} / \mathrm{N}_{p}$ are $\mathrm{p}$-reduced (Lemma 2). If they are actually (torsionfree)-by-(a p'-group)-by-finite, then $\mathrm{G} / \mathrm{N}_{p}$ embeds into $\mathrm{GL}(\mathrm{n}, \mathrm{J})$ for some integer $\mathrm{n}$ and $\mathrm{J}$ the integers localized at $\mathrm{p}$. Thus $\mathrm{G} / \mathrm{N}_{p}$ is a finite extension of a residually finite p-group and consequently $\mathrm{G}$ is a finite extension of a residually finite nilpotent $\pi$-group. 
Open Access This article is distributed under the terms of the Creative Commons Attribution 4.0 International License (http://creativecommons.org/licenses/by/4.0/), which permits unrestricted use, distribution, and reproduction in any medium, provided you give appropriate credit to the original author(s) and the source, provide a link to the Creative Commons license, and indicate if changes were made.

\section{References}

1. Azarov, D.N.: Some residual properties of soluble groups of finite rank (Russian with English summary). Chebyshevski Sb. 15, 7-18 (2014)

2. Kegel, O.H., Wehrfritz, B.A.F.: Locally Finite Groups. North-Holland Pub. Co., Amsterdam (1973)

3. Lennox, J.C., Robinson, D.J.S.: The Theory of Infinite Soluble Groups. Clarendon Press, Oxford (2004)

4. Robinson, D.J.S.: Finiteness Conditions and Generalized Soluble Groups, vol. 2. Springer, Berlin (1972) 